\title{
SPECIAL REPORTS
}

\section{The Year of 2006 in Electrocardiology}

\author{
Shlomo Stern, M.D. \\ From The Hebrew University of Jerusalem, Jerusalem, Israel
}

A.N.E. $2007 ; 12(2): 158-164$

Highlighting the important contributions to the science of electrocardiology published in peerreviewed Journals during the year $2005^{1}$ prompted the observation that ECG is "still the cardiologist's best friend." 2 In this article, I have tried to test whether this last year's phrase did not melt with last year's snow. By reading the following, our readers will be able to judge.

\section{THE P WAVE}

A detailed analysis of the P-wave morphology in focal atrial tachycardia was done by Kistler et al., ${ }^{3}$ who classified them as positive, negative, isoelectric, or biphasic. The authors prospectively evaluated 30 new consecutive patients with focal atrial tachycardia. Their P-wave algorithm correctly identified the site of tachycardia origin in $93 \%$. Lead $\mathrm{V}_{1}$ was the most useful in identifying the likely anatomic site of the origin of the focal atrial tachycardia.

\section{THE QRS COMPLEX}

The prognostic power of computer-measured QRS duration was evaluated by Desai et al. ${ }^{4}$ in a general medical population. The QRS duration score was shown to be a strong independent predictor of cardiovascular mortality: for every $10-\mathrm{ms}$ increase in QRS duration, there was an $18 \%$ increase in cardiovascular risk. The results were similar in patients with an abnormal ECG, with BBB and with paced rhythms. Similar conclusions were reached by Bauer et al. ${ }^{5}$ in post-MI patients in whom a prolonged QRS duration ( $\geq 120 \mathrm{~ms}$ ) showed the highest association with total and late mortality, but not with sudden death and serious arrhythmic events.

Fragmented QRS (fQRS) was defined as the presence of an additional $\mathrm{R}$ wave, notching in the $\mathrm{S}$ wave, or $>1 \mathrm{R}^{\prime}$ in two contiguous leads. ${ }^{6}$ Patients with typical $\mathrm{BBB}$ or incomplete right $\mathrm{BBB}$ were excluded by study design. This study confirmed by using myocardial perfusion imaging that the presence of fQRS on standard 12-lead ECG was more accurate than identification of $Q$ waves, as a valuable sign for prior MI and scar.

In the HERO-2 trial, ${ }^{7}$ Wong et al. studied the influence of different BBB types on prognosis. Right $\mathrm{BBB}$ accompanying anterior $\mathrm{MI}$ at presentation and newly developed right or left BBB early after fibrinolytic therapy were found to be independent predictors of high 30-day mortality. The authors suggested that these ECG features should be considered in risk stratification to identify high-risk patients. In the same trial ${ }^{8}$ on multivariable analyses, an increasing QRS duration by $20-\mathrm{ms}$ increments was associated with increasing 30-day mortality in both right $\mathrm{BBB}$ groups; patients with QRS duration $\geq 160 \mathrm{~ms}$ had a higher 30 -day mortality than those with QRS duration $<160 \mathrm{~ms}$. Also, this trial showed that initial $Q$ waves accompanying ST elevation worsened 30-day mortality. ${ }^{9}$

Late potentials were detected in $62 \%$ of postventriculotomy patients with congenital heart disease by Perloff et al., ${ }^{10}$ who stressed the increased risk for monomorphic VT in these patients. 


\section{THE QT INTERVAL}

In the Rotterdam study, a "dose-response effect" was seen by Straus et al. ${ }^{11}$ between QTc duration and the risk of SCD in the age group 55-68 years and those $>68$ years. An abnormal QTc prolongation, $>450 \mathrm{~ms}$ for men and $>470 \mathrm{~ms}$ for women, was an independent risk factor for SCD. Two-thirds of cases with SCD were shown to have an abnormally prolonged QTc interval. Hiromoto et al. ${ }^{12}$ demonstrated that various indices of beat-to-beat QT interval variability that reflected the infarction site in frequency domain correlated with LV function, but the indices of the RR intervals in frequency domain did not correlate with LV function. This phenomenon may be a result of autonomic imbalance toward sympathetic over activity or vagal withdrawal.

An effect on the QT interval was among the ECG parameters in humans relevant to a decreased arrhythmic risk. Tuna and other broiled or baked fish consumption was associated with lower heart rate, slower atrioventricular conduction, and a substantially lower likelihood of a prolonged QT. ${ }^{13}$

\section{THE T WAVE}

T-wave morphology analysis by Linna et al. ${ }^{14}$ demonstrated that KCNH2 K897T polymorphism was associated with the total cosine R-to-T (TCRT), which reflects the wave from direction between depolarization and repolarization. Subjects with a less common genotype, KT or TT, have smaller TCRT, which reflects dyssynchrony between depolarization and repolarization and may increase their vulnerability to arrhythmic events.

Neurogenic $\mathrm{T}$ wave appearance preceded the clinical acute stroke picture in a patient described by Lindberg and Jauch. ${ }^{15}$ ECG revealed deep, symmetric $\mathrm{T}$-wave inversions, consistent with neurogenic $\mathrm{T}$ waves; the patient developed only 50 minutes after this initial ECG, left-sided hemiplegia, facial droop, eye deviation, and hemineglect.

\section{THE LONG QT SYNDROME}

In LQTS patients, there is a considerable variability in QTc measures in serial follow-up ECGs and the maximum QTc interval provides incremental prognostic information beyond the baseline measurement. Goldenberg et al. ${ }^{16}$ suggested that risk stratification in LQTS patients should include follow-up ECG data of both symptomatic and asymptomatic patients. The detection of QTc of $>500 \mathrm{~ms}$ at any time during follow-up identified patients at high risk for arrhythmic events. The authors stressed the importance of the maximum QTc interval recorded before the age of 10 .

The ECG patterns of the arrhythmia onset and symptom triggers in congenital LQT syndrome were studied by Tan et al. ${ }^{17}$ Pause dependence was predominant in LQT2 but was rare or absent in LQT1; in the latter torsades de pointes started without significant changes in the duration of the preceding RR intervals. The different arrhythmia mechanisms may explain why pause-preventing pacemakers may be required in LQT2, while Badrenergic blockers as single treatment are effective in LQT1.

Vitasalo et al. ${ }^{18}$ proved with the use of 24 -hour ECG recordings that in patients with LQT1 betablockers at heart rates of $>85 \mathrm{bpm}$ shortened both the diurnal maximal $\mathrm{T}$-wave peak to $\mathrm{T}$-wave end interval and the maximal ratio between late and early T-wave peak amplitude, which are ECG counterparts of transmural dispersion of repolarization and early afterdepolarizations, respectively. These observations are in concert with the clinical experience of using beta-blocking medication for the reduction of cardiac events in LQT1 patients.

The predictive power of the QTc interval in all ECG leads was studied by Moenning et al. ${ }^{19}$ in 200 consecutive genotyped LQTS family members with the purpose of using receiver operating curve analysis for identifying mutation carriers and highrisk LQTS patients. Additionally, the risk for events (syncope and sudden cardiac arrest) was calculated for QTc decile in all individuals. The predictive power was highest in lead II and lead $\mathrm{V}_{5}$ for identifying carriers in LQTS families and were optimal for risk stratification.

LQTS contributes to sudden infant death syndrome. Quaglini et al. $^{20}$ analyzed the costeffectiveness of a nationwide program of neonatal ECG screening and demonstrated that the program was cost-effective and allowed the early identification of still asymptomatic infants with LQTS.

In children with LQTS an ambulatory 12-lead system demonstrated macroscopic T-wave alternans, intermittent extreme QT prolongation, and $\mathrm{T}$-wave notchings and in those suspected for tachyarrythmia, several SVTs and VTs. ${ }^{21}$ 


\section{SHORT QT SYNDROME}

Giusetto et al. ${ }^{22}$ reinforced the concept that SCD is the most relevant clinical manifestation of short QT syndrome and that these events may occur in infants and other children. Since ICD implantation during childhood remains a technical challenge in a very young short QT syndrome patient with a highrisk profile, oral quinidine may represent a bridge to ICD implant later in life.

\section{T-WAVE ALTERNANS}

An upsurge in TWA was demonstrated by Shusterman et al. ${ }^{23}$ preceding VT. TWA increased by $10 \% 30$ minutes before and by $25 \% 10$ minutes before the VT started and was not attributable to an increase in heart rate. The specificity of the upsurge in TWA before VT was, however, not proved in this article.

In a relatively low-risk post-MI population with preserved ejection fraction a positive microvolt TWA test was the most significant predictor on multivariate analysis for SCD or life-threatening arrhythmic event and had the highest sensitivity and negative predictive value for these events. ${ }^{24}$ According to the authors, microvolt TWA can be used for risk stratification in this low-risk population. In the study of Kaufman et al. ${ }^{25}$ in patients with left ventricular dysfunction even an "indeterminate" microvolt TWA when due to ectopy, unsustained microvolt TWA, or low exercise heart rate, had similar to a positive test prognostic significance for death or sustained ventricular arrhythmia. Similarly, in patients with ischemic cardiomyopathy, a positive or indeterminate microvolt TWA test was an independent predictor of arrhythmic and allcause mortality with a modest predictive value, as shown by Chow et al. ${ }^{26}$

\section{ISCHEMIA/INFARCTION DIAGNOSIS}

The precise ECG diagnosis of acute MI was the subject of several recent investigations. In a multicenter study, the prevalence of failure to identify high-risk ECG findings (significant ST-segment depressions, ST-segment elevations, or T-wave inversions) in emergency departments was studied by Masoudi et al. ${ }^{27}$ In patients with confirmed acute MI on presentation, the failure rate was $12 \%$. Rates of missed ECG findings by hospitals ranged from $5.6 \%$ to $15.1 \%$. Missed high-risk findings were less common among patients with ST segment eleva- tion than for those with T-wave inversion. The in hospital mortality of the patients with missed highrisk ECG findings was $7.9 \%$ compared with $4.9 \%$ among those without missed findings.

In ST-elevation acute MI patients in the MONAMI study, ${ }^{28}$ continuous ST-monitoring was initiated in the pre-hospital phase and continued during and 90 minutes following PCI. Patient classification based on the ST monitoring result was useful for risk stratification at time of PCI into groups with low, intermediate, and high risk profile. Westerhout et al. ${ }^{29}$ demonstrated that the degree of STsegment depression on 12-lead baseline ECG was the highest contributor to the prediction of 30-day and 1-year mortality and 30-day death/MI in a contemporary sample of 7800 non-ST-elevation ACS patients, beyond troponin $\mathrm{T}$, creatinine clearance, $\mathrm{N}$-terminal pro-brain natriuretic peptide, heart rate, and age.

The concordance between the location of an acute myocardial infarction by the ECG patterns and by cardiac MR showed that abnormally increased $\mathrm{R}$ waves, the Q-wave equivalent, in leads $\mathrm{V}_{1}$ and $\mathrm{V}_{2}$ indicate a lateral-wall $\mathrm{MI}$ and that abnormal $\mathrm{Q}$ waves in leads aVL and I without a $\mathrm{Q}$ wave in lead $\mathrm{V}_{6}$ indicate a mid-anterior MI. ${ }^{30}$ Accordingly, the terms "posterior" and "high lateral" MI are incorrect when applied to these patterns and it is suggested that these should be changed to lateral-wall and "mid-anterior wall" MI, respectively.

In patients with inferior ST-elevation MI V4R yielded information, which led to an enhanced use of fibrinolytic therapy, as shown by Harju et al. ${ }^{31}$

Unipolar intracoronary ECG recording from the angioplasty catheter guidewire has been shown by Balian et al. ${ }^{32}$ to be more sensitive and reliable to detect myocardial injury than standard ECG. By obtaining tracings before and after PCI, the authors determined the degree of ST-segment elevation or depression compared with the baseline intracoronary ST-segment. Patients who had a significant ST-segment shift of $\geq 1 \mathrm{~mm}$ were more likely to have myonecrosis, as measured either by troponin or CK-MB and had a trend toward a greater number of hard clinical events. Of five patients who suffered a fatal or nonfatal MI, four had an ST-segment shift of $\geq 1 \mathrm{~mm}$.

In a case control study with a first ST-elevation MI, in VF survivors cumulative ST-segment elevation was shown to be significantly greater preceding the $\mathrm{VF}$ than in controls, whereas infarct size was similar in the two groups, according to an analysis 
by Dekker et al. of 330 primary VF survivors and of 372 controls. ${ }^{33}$

\section{ARRTHYTHMIA DETECTION}

New information derived by Rosman et al. ${ }^{34}$ analyzing patients with ICDs showed that a VPB initiated the VT in 58 episodes (92\%), 1 episode was initiated by a supraventricular tachycardia, and 4 episodes had sudden onset.

The prematurity ratio was significantly higher in patients with ischemic cardiomyopathy as compared to patients with nonischemic cardiomyopathy. This may represent different mechanisms of VT initiation in patients with ischemic versus nonischemic heart disease.

In apparently healthy persons without history or clinical monifestasionsof heart disease, 2-minute ECG demonstrated VBPs in $6.2 \%$ in over 15,000 examinees in a study by Massing et al. ${ }^{35}$ After a follow-up of $>10$ years, the mortality was $>3$ times greater for those with VPBs compared with those without VPBs. Similar results were reported by Sajadieh et al. ${ }^{36}$ who found that single VPBs on a standard ECG were significant prediction of frequent VPBs and an independent predictor of allcourse mortality and acute MI. Thus, VPBs even in apparently healthy individuals warrant awareness and meticulous risk assessment.

\section{THE BRUGADA SYNDROME}

In the Brugada syndrome type 1, i.e., the "covedtype" ST-segment is mandatory for the diagnosis. In 43 consecutive patients of Brugada syndrome, Weltman et al. ${ }^{37}$ found in only one patient a consistent type 1 ECG, whereas in about half of them it appeared and disappeared transiently during the follow-up, and in the others was only inducible by drugs. Thus, a patient's risk for future events may be underestimated if it based only on an initial nondiagnostic ECG, and repetitive ECG recordings therefore seem to be mandatory. Daily fluctuations in the magnitude of the right precordial ST-segment elevations and the appearance of late potentials might possess predictive power for future events. ${ }^{38}$

Unmasking the Brugada type 1 ECG phenotype as a result of amiodarone therapy was reported by Paul et al. ${ }^{39}$ in a patient with atrial fibrillation and poorly controlled ventricular rate. An intravenous amiodarone infusion was commenced that not only restored sinus rhythm within 12 hours, but also, unmasked the classic features of type 1 Brugada syndrome. Discontinuation led to a resolution of the ECG changes.

\section{EXERCISE ECG}

For years, most experts have advocated that upsloping ST-segment depression during exercise testing does not indicate ischemia. Recently, ${ }^{40}$ retrospective analysis of 621 exercises tests on patients, who have had nuclear scintigraphy and coronary angiograms within a 3-month period, showed that $20 \%$ of patients with this configuration had a large area of reversible ischemia on the gated SPECT acquisition and $28 \%$ had triple-vessel disease on coronary angiography. Consequently, when upsloping ST changes were considered abnormal, the sensitivity of the exercise EKG increased to $82 \%$ when compared to the gated SPECT imaging and to $77 \%$ when using the angiogram as the gold standard, while the specificity remained about the same.

Kligfield and Lauer ${ }^{41}$ in a recent report stressed that beyond recognizing "simple" ST depression, the measuring of heart rate adjustment of the ST depression, QRS duration and amplitude, QT- and $\mathrm{T}$ wave changes, ST recovery loops and hysteresis, improve the diagnostic capability of exercise testing. High frequency components of the QRS complex that are not usually represented in the routine ECG tracing, between 150 and $250 \mathrm{~Hz}$, have been shown by Abboud and Zlochiver ${ }^{42}$ to decrease in the presence of acute ischemia.

\section{HYPERTENSION}

In hypertensive patients, a strain pattern, defined as a down-sloping convex ST segment with inverted asymmetrical T-wave opposite the QRS axis in lead $\mathrm{V}_{5}$ or $\mathrm{V}_{6}$, was shown by Okin et al. ${ }^{43}$ to represent an increased risk of developing heart failure and of dying as a result of heart failure. This was found also even in the setting of aggressive blood pressure lowering, suggesting that more aggressive therapy may be warranted in hypertensive patients with a strain pattern.

\section{PROGNOSTIC VALUE OF ECG}

The prognostic information from the 12-lead ECG in noncardiac surgery candidates with coronary artery disease was studied by Jeger et al. ${ }^{44}$ $\mathrm{ST}$ depression and faster RR intervals remained 
independent predictors of all-cause mortality after adjustment for clinical baseline findings. Faster heart rate was also an independent predictor of major adverse cardiac events at 2 years.

The importance of a prehospital 12-lead ECG was demonstrated by showing that it reduces the median time to fibrinolytic therapy in the hospital by 32 to $62 \mathrm{~min}^{45}$

\section{GENDER DIFFERENCES}

In contrast to the widespread conception that the ECG is of limited utility in women, Okin et al. $^{46}$ stressed recently that in female patients the value of the ECG for risk stratification was similar to that in males. Rautaharaju et al. ${ }^{47}$ studied five ECG variables in men and women and found them to be equally significant mortality predictors in both genders. The same group of investigators described the repolarization abnormalities in postmenopausal women, which predict coronary events and death. ${ }^{48}$

In women $\geq 90$ years young, presenting for any reason, a normal ECG tracing was found more frequently than in men in the similar age group $16 \%$ vs $2 \%) .{ }^{49}$

\section{ECG IN CARDIOMYOPATHY}

In restrictive cardiomyopathy due to amyloidosis a striking ECG evolution was shown by Leeson et al. ${ }^{50}$ : ST-segment change, reduced QRS amplitude, increased $\mathrm{P}$ wave width and amplitude, and progressive increase in PR interval. P-wave morphology demonstrated additional significant atrial involvement. Restrictive cardiomyopathy leads to bi-atrial enlargement and increased $\mathrm{P}$ wave amplitude and width. Furthermore, atrial amyloid deposition delays atrial conduction and thereby increases the PR interval and broadens the $\mathrm{P}$ wave. The bi-atrial pathologies were clearly demonstrated in this patient by cardiovascular magnetic resonance imaging.

The ECG findings in 102 hypertrophic cardiomyopathy patients were compared with the results of cardiac magnetic resonance by Dumont et al. ${ }^{51} \mathrm{Ab}$ normal $\mathrm{Q}$ waves were associated with greater upper anterior septal thickness and increased ratios of upper anterior septum to mean inferolateral, anterolateral, apical, and right ventricular wall thickness. Wider $\mathrm{Q}$ waves, conduction disturbances, and absent septal $\mathrm{Q}$ waves were associated with late- enhancement. The depth of negative $\mathrm{T}$ waves was related to craniocaudal asymmetry and apical lateenhancement.

\section{ECG IN MYOCARDITIS}

In parvovirus B19 myocarditis patients frequently present predominantly with severe chest pain of acute onset in a manner similar to that of myocardial infarction; most had typical subepicardial late gadolinium enhancement in the lateral wall. This combination of ischemia and inflammation explains, according to Mahrholdt et al., ${ }^{52}$ the infarct-like ECG and elevated troponin level.

\section{CONCLUSIONS}

Even without reporting here on dramatic advances during 2006 in electrocardiology, in view of the uninterrupted flow of hundreds of new, original, and inventive articles, I am confident to say that ECG is today the most widely used important method in cardiology. The reviewed 50 outstanding innovative articles prove the relevance of ECG not only for diagnosis, but also for decision making regarding the therapies available today and for identifying the individual at high risk for cardiac events and for sudden cardiac deaths.

Acknowledgments: The excellent secretarial help of Mrs. Estelle Rachamim-Rayman is greatly acknowledged.

\section{REFERENCES}

1. Stern S. The year 2005 in electrocardiology. Ann Noninvasive Electrocardiol 2006;11:187-193.

2. Stern S. Electrocardiogram: Still the cardiologist's best friend. Circulation 2006;113:e753-e756.

3. Kistler PM, Robert-Thomson KC, Haqqani HM, et al. Pwave morphology in focal atrial tachycardia: Development of an algorithm to predict the anatomic site of origin. J Am Coll Cardiol 2006;48:1010-1017.

4. Desai AD, Yaw TS, Yamazaki $T$, et al. Prognostic significance of quantitative QRS duration. Am J Med 2006;119:600-606.

5. Bauer A, Watanabe MA, Barthel P, et al. QRS duration and late mortality in unselected post-infarction patients of the revascularization era. Eur Heart J 2006;27:427-433.

6. Das MK, Khan B, Jacobs $S$, et al. Significance of a fragmented QRS complex versus a $Q$ wave in patients with coronary artery disease. Circulation 2006;113:2495-2501.

7. Wong CK, Stewart RAH, Gao W, et al. Prognostic differences between different types of bundle branch block during the early phase of acute myocardial infarction: Insights from the Hirulog and Early Reperfusion or Occlusion (HERO)-2 trial. Eur Heart J 2006;27:21-28.

8. Wong CK, Gao W, Stewart RAH, et al. Risk stratification of patients with acute anterior myocardial infarction and right bundle-branch block: Importance of QRS duration and early 
ST-segment resolution after fibrinolytic therapy. Circulation 2006;114:783-789.

9. Wong CK, Gao W, Raffel OC, et al. Initial $Q$ waves accompanying ST elevation at presentation of myocardial infarction and 30-day mortality in patients given streptokinase therapy: An analysis from HERO-2. Lancet 2006;367:20612067.

10. Perloff JK, Middlekauf HR, Child JS, et al. Usefulness of post-ventriculotomy signal averaged electrocardiograms in congenital heart disease. Am J Cardiol 2006;98:1646-1651.

11. Straus SMJM, Kors JA, De Bruin ML, et al. Prolonged QTc interval and risk of sudden cardiac death in a population of older adults. J Am Coll Cardiol 2006;47:362-367.

12. Hiromoto K, Shimizu H, Mine T, et al. Correlation between beat-to-beat QT interval variability and impaired left ventricular function in patients with previous myocardial infarction. Ann Noninvasive Electrocardiol 2006;11:299-305.

13. Mozaffarian D, Prineas RJ, Stein PK, et al. Dietary fish and n-3 fatty acid intake and cardiac electrocardiographic parameters in humans. J Am Coll Cardiol 2006;48:478-484.

14. Linna EH, Perkiomaki JS, Karsikas M, et al. Functional Significance of KCNH2 (HERG) K897T polymorphism for cardiac repolarization assessed by analysis of T-wave morphology. Ann Noninvasive Electrocardiol 2006;11:57-62.

15. Lindberg DM, Jauch EC. Neurogenic T waves preceding acute ischemic stroke. Circulation 2006;114:e369-e370.

16. Goldenberg I, Mathew J, Moss AJ, et al. Corrected QT variability in serial electrocardiograms in long QT syndrome: The importance of the maximum corrected QT for risk stratification. J Am Coll Cardiol 2006;48:1047-1052.

17. Tan HL, Bardai A, Shimizu W, et al. Genotype-specific onset of arrhythmias in congenital long QT syndrome: Possible therapy implications. Circulation 2006;114:20962103.

18. Vitasalo M, Oikarinen L, Swan $\mathrm{H}$, et al. Effects of betablocker therapy on ventricular repolarization documented by 24 -h electrocardiography in patients with type 1 longOT-syndrome. J Am Coll Cardiol 2006:48:747-753.

19. Moennig G, Eckardt L, Wedekind H, et al. Electrocardiographic risk stratification in families with congenital long QT syndrome. Eur Heart J 2006;27:2074-2080.

20. Quaglini S, Rognoni C, Spazzolini C, et al. Cost-effectiveness of neonatal ECG screening for the long QT syndrome. Eur Heart J 2006;27:1824-1832.

21. Emmel M, Sreeram N, Schickendantz S, et al. Experience with an ambulatory 12-lead Holter recording system for evaluation of pediatric dysrhythmias. J Electrocardiol 2006;39:188-193.

22. Giustetto C, Di Monte F, Wolpert C, et al. Short QT syndrome: Clinical findings and diagnostic therapeutic implications. Eur Heart J 2006;27:2440-2447.

23. Shusterman V, Goldberg A, London B. Upsurge in T-wave alternans and nonalternating repolarization instability precedes spontaneous initiation of ventricular tacharrythmias in humans. Circulation 2006;113:2880-2887.

24. Ikeda T, Hideaki $Y$, Sugi $K$, et al. Predictive value of microvolt T-wave alternans for sudden cardiac death in patients with preserved cardiac function after acute myocardial infarction: Results of a collaborative cohort study. J Am Coll Cardiol 2006;48:2268-2274

25. Kaufman ES, Bloomfield DM, Steinman RC, et al. "Indeterminate" microvolt T-wave alternans tests predict high risk of death or sustained ventricular arrhythmias in patients with left ventricular dysfunction. J Am Coll Cardiol 2006;48:1399-1404.

26. Chow T, Kereiakes DJ, Bartone $\mathrm{C}$, et al. Prognostic utility of microvolt $\mathrm{T}$-wave alternans in risk stratification of patients with ischemic cardiomyopathy. J Am Coll Cardiol 2006;47:1820-1827.
27. Masoudi FA, Magid DJ, Vinson DR, et al. Implications of the failure to identify high-risk electrocardiogram findings for the quality of care of patients with acute myocardial infarction: Results of the emergency department quality in myocardial infarction (EDQMI) study. Circulation 2006:114:1565-1571.

28. Terkelsen CJ, Norgaard BL, Lassen JF, et al. Potential significance of spontaneous and interventional ST- changes in patients transferred for primary percutaneous coronary intervention: Observations from the ST-MONitoring in acute myocardial infarction study (The MON AMI study). Eur Heart J 2006;27:267-275.

29. Westerhout $\mathrm{CM}, \mathrm{Fu} \mathrm{Y}$, Lauer MS, et al. Short and longterm risk stratification in acute coronary syndromes: The added value of quantative ST-segment depression and multiple biomarkers. J Am Coll Cardiol 2006;48:939947.

30. Bayes De Luna A, Wagner G, Birnbaum Y, et al. A New terminology for left ventricular walls and location of myocardial infarcts that present $Q$ wave based on the standard of cardiac magnetic resonance imaging. Circulation 2006;114:1755-1760.

31. Harju JA, Eskola MJ, Huhtala H, et al. Recording lead V4R is associated to enhanced use of fibrinolytic therapy in acute myocardial infarction. J Electrocardiol 2006;39:368.el368.e5.

32. Balian V, Galli M, Marcassa C, et al. Intracoronary STsegment shift soon after elective percutaneous coronary intervention accurately predicts periprocedural myocardial injury. Circulation 2006;114:1948-1954.

33. Dekker LRC, Bezzina CR, Henriques JPS, et al. Familial sudden death is an important risk factor for primary ventricular fibrillation: A case-control study in acute myocardial infaction patients. Circulation 2006;114:1140-1145.

34. Rosman J, Hanon S, Shapiro M, et al. Triggers of sustained monomorphic ventricular tachycardia differ among patients with varying etiologies of left ventricular dysfunction. Ann Noninvasive Electrocardiol 2006;11:113-117.

35. Massing MW, Simpson RJ, Rautaharju PM, et al. Usefulness of ventricular Premature complexes to predict coronary heart disease events and mortality (from the Atherosclerosis Risk in Communities cohort). Am J Cardiol 2006;98:16091612 .

36. Sajadieh A, Nielsen OW, Rasmussen V, et al. Ventricular arrhythmias and risk of death and acute myocardial infarction in apparently health subjects of age 55 years. Am J Cardiol 2006:97:1351-1357.

37. Veltmann C, Schimpf R, Echternach $C$, et al. A prospective study on spontaneous fluctuations between diagnostic and non-diagnostic ECGs in Brugada syndrome: Implications for correct phenotyping and risk stratification. Eur Heart J 2006;27:2544-2552.

38. Tatsumi $H_{\text {, Takagi }} M$, Nakagawa $E$, et al. Risk stratification in patients with Brugada syndrome: Analysis of daily fluctuations in 12-lead electrocardiogram (ECG) and signalaveraged electrocardiogram (SAECG). J Cardiovasc Electrophysiol 2006;17:705-711.

39. Paul G, Yusuf S, Sharma S. Unmasking of the Brugada Syndrome Phenotype during the acute phase of amiodarone infusion. Circulation 2006;114:e489-e491.

40. Polizos G, Ellestad MH. The value of upsloping ST depression in diagnosing myocardial ischemia. Ann Noninvasive Electrocardiol 2006;11:237-239.

41. Kligfield P, Lauer MS. Exercise electrocardiogram testing: Beyond the ST segment. Circulation 2006;114:20702082.

42. Abboud S, Zlochiver S. High-frequency QRS electrocardiogram for diagnosing and monitoring ischemic heart disease. J Electrocardiol 2006;39:82-86. 
43. Okin PM, Devereux RB, Nieminen MS, et al. Electrocardiographic strain pattern and prediction of new-onset congestive heart failure in hypertensive patients. The Losartan Intervention for Endpoint Reduction in Hypertension (LIFE) Study. Circulation 2006;113:67-73.

44. Jeger RV, Probst C, Arsenic R. Long-term prognostic value of the preoperative 12-lead electrocardiogram before major noncardiac surgery in coronary artery disease. Am Heart J 2006; 151:508-513.

45. Garvey JL, MacLeod BA, Sopko G, et al. Pre-hospital 12-lead electrocardiography programs. J Am Coll Cardiol 2006:47:485-491.

46. Okin PM. Electrocardiography in women. Taking the initiative. Circulation 2006;113:464-466.

47. Rautaharju PM, Ge S, Nelson JC, et al. Comparison of mortality risk for electrocardiographic abnormalities in men and women with and without coronary heart disease (from the Cardiovascular Health Study). Am J Cardiol 2006;97:309315 .
48. Rautaharju PM, Kooperberg C, Larson J, et al. Electrocardiographic abnormalities that predict coronary heart disease events and mortality in postmenopausal women: The women's health initiative. Circulation 2006;113:471480.

49. Kelley GP, Stellingworth MA, Broyle S, et al. Electrocardiographic findings in 888 patients 90 years of age. Am J Cardiol 2006:98:1512-1514.

50. Leeson CP, Myerson SG, Walls GB, et al. Atrial pathology in cardiac amyloidosis: Evidence from ECG and cardiovascular magnetic resonance. Eur Heart J 2006;27: 1670.

51. Dumont CA, Monserrat $\mathrm{L}$, Soler $\mathrm{R}$, et al. Interpretation of electrocardiographic abnormalities in hypertropic cardiomyopathy with cardiac magnetic resonance. Eur Heart J 2006;27:1725-1731.

52. Mahrholdt $\mathrm{H}$, Wagner A, Deluigi CC, et al. Presentation patterns of myocardial damage, and clinical course of viral myocarditis. Circulation 2006;114:1581-1590. 